\title{
Análise de fluxo de potência através de métodos numéricos
}

\author{
Power flow analysis using numerical methods
}

\author{
Bruno Pereira do Nascimento ${ }^{1}$, Caison Rodrigues Ramos ${ }^{2}$ e Aline Brum Loreto \\ ${ }^{1}$ Universidade Federal de Santa Maria, Cachoeira do Sul, Brasil \\ bruinonasc@outlook.com \\ ${ }^{2}$ Universidade Federal de Santa Maria, Cachoeira do Sul, Brasil \\ caisonrr@hotmail.com \\ ${ }^{3}$ Universidade Federal de Santa Maria, Cachoeira do Sul, Brasil \\ aline.loreto@ufsm.br
}

\section{Resumo}

O Sistema Elétrico de Potência tem como função básica fornecer energia elétrica com qualidade e quando solicitada. Para que isso seja possível são necessárias algumas análises do sistema, dentre elas a Análise do Fluxo de Potência. Essa análise é importante para delineação dos sistemas de potência, assim como também, na definição das melhores condições de operação, controle e supervisão dos sistemas existentes. O sistema é modelado da seguinte forma: Geradores, Cargas, Reatores e Capacitores que são ligados entre um nó (barra) qualquer e o nó terra, já as linhas de transmissão e transformadores são ligadas entre dois nós quaisquer. Assim será gerada através de análise nodal a matriz de admitância do sistema, onde este problema é resolvido através de métodos numéricos. Um dos objetivos deste trabalho é a análise de fluxo de potência de um sistema com auxílio de métodos numéricos. Outro objetivo é verificar a exatidão dos resultados, com soluções obtidas pelos métodos de Eliminação de Gauss, Fatoração LU, Gauss Seidel e Método de Crout, implementados em linguagem C. A análise da exatidão dos resultados deu-se através do erro relativo em comparação com os resultados obtidos pelo software MatLab.

Palavras-chave: Fluxo de potência; Métodos numéricos; Linguagem C

\section{Abstract}

The basic function of the Electric Power System is to supply electrical energy with quality and when requested. For this to be possible some analysis of the system is required, among them Power Flow Analysis. This analysis is important for the delineation of the power systems, as well as in the definition of the best conditions of operation, control and supervision of the existing systems. The system is modeled as follows: Generators, Loads, Reactors and Capacitors are connected between any node and the ground node, since the transmission lines and transformers are connected between any two nodes. Thus, the admittance matrix of the system will be generated through nodal analysis that will be solved by numerical methods. One of the objectives of this work aims to perform the power flow analysis of a system with the aid of numerical methods. Another objective is as well as to verify the accuracy of the results, with solutions obtained by the methods of Gauss Elimination, LU Factoration, Gauss Seidel and Crout Method, implemented in C language. The analysis of the accuracy of the results occurred through the relative error in comparison to the results obtained by MatLab software.

Keywords: Power flow; Numerical methods; C language 


\section{Introdução}

O fluxo de potência consiste na determinação das tensões nas barras do sistema, do fluxo que é transmitido pelas linhas, dentre outras grandezas de interesse. Permite determinar o estado operativo do sistema, verificando se está operando corretamente, para poder corrigir ou prevenir contingências (MONTICELLI, 1983).

Este trabalho tem o objetivo de realizar uma análise do fluxo de potência ativa de um sistema com auxílio de métodos numéricos, que será realizado em matemática real - aritmética e sistemas reais- Com a verificação da ocorrência de maior exatidão nos resultados obtidos, e escrever códigos/rotinas de programas computacionais livres que sejam capazes de obter resultados de com qualidade considerável.

Para tal, utilizam-se os métodos de Eliminação de Gauss, Fatoração LU, Gauss Seidel e Crout (CHAPRA e CONALE, 2008). Sendo estes, códigos programados a partir da linguagem de programação C. Os resultados obtidos foram comparados com o software comercial MatLab, possibilitando assim a comparação/validação e o cálculo do erro relativo dos métodos.

\section{Referencial Teórico}

\subsection{Fluxo de Potência}

A análise do fluxo de potência consiste em estabelecer um estado na rede, com a distribuição dos fluxos e outras variáveis de interesse do problema. (MONTICELLI, 1983). Nos sistemas de potência, os elementos são ligados de duas maneiras diferentes: a primeira entre barras e a segunda entre a barra de referência e uma barra qualquer. Os geradores e as cargas são tidos como parte externa e modelados como injeções constantes de potência nas barras da rede. A parte interna da rede, formada pelos demais componentes é tida como um conjunto de circuitos passivos e modelada por meio da matriz de admitância de barra (MONTICELLI, 1983).

Quatro grandezas estão associadas a cada barra da rede, este representado pelo sub índice k, onde:

$\mathrm{V}_{\mathrm{k}}$, que é o módulo da tensão na barra k;

$\theta_{\mathrm{k}}$, é o ângulo da tensão na barra k;

$\mathrm{P}_{\mathrm{k}}$ : Potência ativa líquida injetada na barra $\mathrm{k}$;

$\mathrm{Q}_{\mathrm{k}}$ : Potência reativa líquida injetada na barra $\mathrm{k}$.

Dependendo de como estas grandezas são tratadas no problema do fluxo de potência, são então definidos os tipos de barras:

- Barra de carga ou PQ: Conhecem-se as grandezas $P_{k}$ e $Q_{k}$ calculam-se $V_{k}$ e $\theta_{k}$.

- Barra de tensão controlada ou PV: Conhecidos $P_{k}$ e $V_{k}$ calculam-se $Q_{k} e \theta_{k}$.

- Barra remota ou PQV: Conhecidos $P_{k}, Q_{k}$ e $V_{k}$, calcula-se $\theta_{k}$ (MONTICELLI, 1983).

O fluxo de potência ativa no sistema é aproximadamente proporcional a abertura angular, e se desloca no sentido dos ângulos maiores para os ângulos menores. A relação entre fluxo de potência ativa e aberturas angulares é do mesmo tipo da existente entre os fluxos de corrente e as quedas de tensões em um circuito de corrente contínua. Essa propriedade possibilita o desenvolvimento de um modelo aproximado chamado de fluxo de carga CC, que permite estimar o fluxo de potência ativa em um sistema (MONTICELLI, 1983).

$\mathrm{O}$ fluxo de potência $\mathrm{P}_{k m}$ em um sistema de potência é dado por:

$$
P_{k m}=V_{k}^{2} g_{k m}-V_{k} V_{m} g_{k m} \cos \theta_{k m}-V_{k} V_{m} b_{k m} \operatorname{sen} \theta_{k m},
$$


onde o sub índice km representa a identificação dos pontos das barras $K$ e $m$.

O fluxo no extremo oposto da linha é dado por:

$$
P_{k m}=V_{k}^{2} g_{k m}-V_{k} V_{m} g_{k m} \cos \theta_{k m}+V_{k} V_{m} b_{k m} \operatorname{sen} \theta_{k m}
$$

As perdas de transmissão na linha são dadas por:

$$
P_{k m}+P_{m k}=g_{k m}\left(V_{k}^{2}+V_{m}^{2}-2 V_{k} V_{m} \cos \theta_{k m}\right.
$$

Se os termos correspondentes as perdas forem desprezadas nas expressões de $\mathrm{P}_{k m}$ e $\mathrm{P}_{m k}$, obtém-se:

$$
P_{k m}=-P_{m k}=-V_{k} V_{m} b_{k m} \operatorname{sen} \theta_{k m}
$$

Se forem feitas as seguintes aproximações:

$$
\begin{aligned}
& V_{k} \cong V_{m} \cong 1 p u, \\
& \operatorname{sen} \theta_{k} \cong \theta_{k}, \\
& b_{k m} \cong-\frac{1}{x_{k m}} .
\end{aligned}
$$

$\mathrm{O}$ fluxo $\mathrm{P}_{k m}$ pode ser aproximado por:

$$
P_{k m}=x_{k m}{ }^{-1} \theta_{k m}=\frac{\theta_{k}-\theta_{m}}{x_{k m}}
$$

Essa expressão ainda pode ser escrita na forma:

$$
P_{k}=\left(\sum_{m \in \Omega_{k}} x_{k m}{ }^{-1}\right) \theta_{k}+\sum_{m \in \Omega_{k}}\left(-x_{k m}{ }^{-1} \theta_{k}\right)
$$

E em representação matricial na forma em (2):

$$
P=B \theta
$$

onde B é a matriz admitância do sistema dada por (3):

$$
B_{k m}=-x_{k m}^{-1} \quad B_{k k}=\sum_{m \in \Omega_{k}} x_{k m}^{-1}
$$

\subsection{Métodos para solução de Sistemas lineares}

Em Métodos Numéricos existem diversos métodos para solução de sistemas os quais podem ser utilizados para resolver os sistemas gerados pela análise do fluxo de potência. Neste trabalho utilizaram-se os métodos de Eliminação de Gauss, Fatoração LU, Gauss Seidel e Método de Crout.

O Método de Eliminação de Gauss foi estudado e implementado pelo cientista Carl Friedrich Gauss. O mesmo zera os elementos abaixo do elemento de pivô, conforme percorre a matriz (CHAPRA e CONALE, 2008).

A Figura 1 apresenta uma matriz triangular superior formada após o método de Eliminação de Gauss. 
Figura 1 - Matriz Triangular superior

$$
\left[\begin{array}{ccccc}
A_{11} & A_{12} & A_{13} & \ldots & A_{1 n} \\
0 & A_{22} & A_{23} & \ldots & A_{2 n} \\
0 & 0 & A_{33} & \ldots & A_{3 n} \\
0 & 0 & 0 & \ldots & A_{4 n} \\
\vdots & \vdots & \ddots & \ddots & \vdots \\
0 & 0 & 0 & \ldots & A_{n n}
\end{array}\right]_{n \times n}
$$

O Método de Fatoração LU, tem como objetivo fatorar a matriz A no produto de duas matrizes L e U, sendo L uma matriz triangular inferior e U uma matriz triangular superior, conforme a Figura 2 (CHAPRA e CONALE, 2008).

Figura 2 - Fatoração LU

$$
\mathbf{A}=\mathbf{L} \mathbf{0}=\underbrace{\left[\begin{array}{cccc}
1 & 0 & \ldots & 0 \\
m_{21} & 1 & \ddots & \vdots \\
\vdots & \ddots & \ddots & 0 \\
m_{n 1} & \ldots & m_{n n-1} & 1
\end{array}\right]}_{\mathbf{L}} \underbrace{\left[\begin{array}{cccc}
a_{11}^{(1)} & \ldots & \ldots & a_{1 n}^{(1)} \\
0 & a_{22}^{(2)} & \ldots & a_{22 n}^{(2)} \\
\vdots & \ddots & \ddots & \vdots \\
0 & \ldots & 0 & a_{n n}^{(n)}
\end{array}\right]}_{\mathbf{U}}
$$

O método de Crout é utilizado na resolução de problemas de sistemas de equações lineares onde a matriz dos coeficientes é tridiagonal. Tal matriz é fatorada em duas matrizes L e U (CHAPRA e CONALE, 2008).

O método de Gauss Seidel é o método interativo mais usado que consiste em isolar o temo da diagonal principal, isolar $\mathrm{x}_{1}$ na primeira equação, $\mathrm{x}_{2}$ na segunda equação, $\mathrm{x}_{\mathrm{n}}$ na n-ésima equação. Uma forma simples de obter aproximações iniciais é supor que elas são todas nulas. Esses zeros podem ser substituídos na equação, que pode ser usada para calcular um novo valor para $x_{1}=b_{1} / a_{11}$. Então, substitui-se esse novo valor de $x_{1}$ junto com a aproximação anterior nula para $x_{3}$ para calcular um novo valor para $\mathrm{x}_{2}$. O processo é repetido para se calcular uma nova estimativa para $\mathrm{x}_{3}$. Então, volta-se para a primeira equação e o procedimento inteiro é repetido até que a solução convirja para valores suficientemente próximos dos valores verdadeiros (CHAPRA e CONALE, 2008).

A Figura 3 apresenta as equações formadas pelo método de Gauss Seidel.

Figura 3 - Gauss Seidel

$$
\left|\begin{array}{rl}
x_{1}= & \frac{1}{a_{11}}\left[b_{1}-a_{12} x_{2}-a_{13} x_{3}-\cdots-a_{1 n} x_{n}\right] \\
x_{2}= & \frac{1}{a_{22}}\left[b_{2}-a_{21} x_{1}-a_{23} x_{3}-\cdots-a_{2 n} x_{n}\right] \\
x_{3}= & \frac{1}{a_{33}}\left[b_{3}-a_{31} x_{1}-a_{32} x_{2}-\cdots-a_{3 n} x_{n}\right] \\
& \vdots \\
x_{n}= & \frac{1}{a_{n n}}\left[b_{n}-a_{n 1} x_{1}-a_{n 2} x_{2}-\cdots-a_{n, n 1} x_{n-1}\right]
\end{array}\right|
$$

\section{Trabalhos relacionados}

Os autores Oliveira e Soares Filho (2003), apresentam um modelo de análise de potência ativa com critério quadrático separável usando métodos de pontos interiores. A abordagem utilizada combina as vantagens da formulação do modelo DC por 
fluxo em redes com a eficiência e robustez dos métodos de pontos interiores. Ela explora em profundidade a estrutura matricial particular do problema, reduzindo o sistema linear a ser resolvido à dimensão do número de barras ou de laços independentes. Em ambos os casos, a matriz resultante é invariante durante as iterações, podendo ser fatorada a priori.São apresentados resultados numéricos envolvendo sistemas testes do IEEE e do sistema elétrico brasileiro.

Baptista et al. (2004) apresentam uma nova abordagem para a resolução do problema de Fluxo de Potência Ótimo. Nesta as restrições canalizadas são tratadas pelo método de barreira logarítmica. Uma função Lagrangiana aumentada é associada ao problema modificado. As condições necessárias de primeira ordem são aplicadas gerando um sistema não-linear que é resolvido pelo método de Newton. Os multiplicadores de Lagrange associados às desigualdades são atualizados pela regra proposta por Hestenes.

\section{Procedimentos Metodológicos}

Foram analisados dois sistemas de tamanhos diferentes para verificar se o tamanho do sistema linear interfere na exatidão dos métodos implementados.

Para a análise do fluxo de potência ativa do sistema, Figura 4, primeiro foi construída a matriz admitância de acordo com (3), que gerou uma matriz $4 \times 4$. A barra 4 foi definida como barra de referência, logo sabe-se que o ângulo $\theta_{4}=0$, assim a linha 4 e a coluna 4 da matriz admitância podem ser desconsideradas formando uma matriz $3 \times 3$. Através da (2) montou-se o sistema a ser resolvido (Figura 5).

Figura 4 - Sistema Elétrico de Potência 4 barras

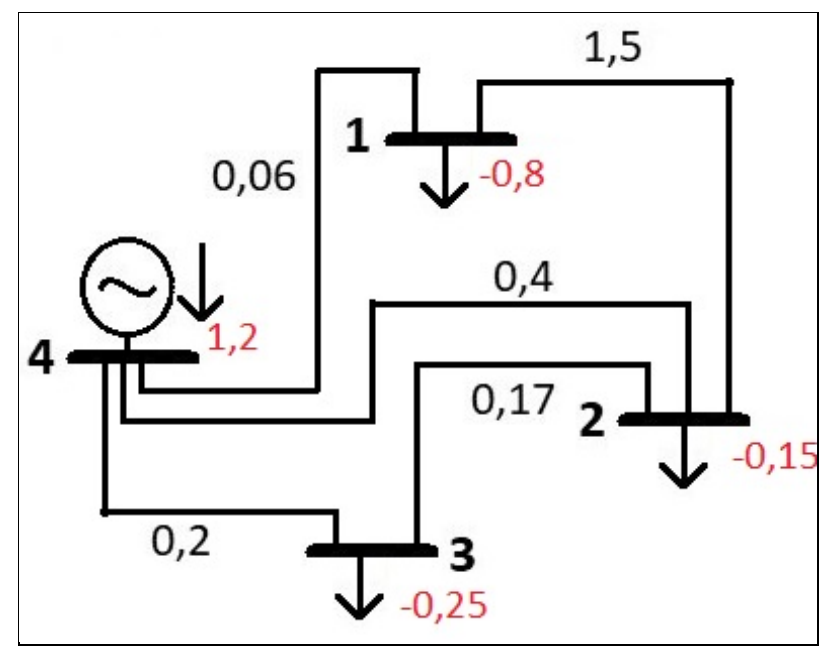

Figura 5 - Sistema linear 4 barras

$\left(\begin{array}{ccc}17,333333 & -0,666666 & 0 \\ -0,666666 & 9,04902 & -5,882353 \\ 0 & -5,882353 & 10,882353\end{array}\right) \cdot\left(\begin{array}{l}\theta_{1} \\ \theta_{2} \\ \theta_{3}\end{array}\right)=\left(\begin{array}{c}-0,8 \\ -0,15 \\ -0,25\end{array}\right)$


A seguir foi feito processo análogo para o sistema, Figura 6, primeiro foi construída a matriz admitância de acordo com (3), que gerou uma matriz 10x10. Mas barra definida como barra de referência foi a barra 1 , logo sabe-se que o ângulo $\theta_{1}=0$, assim a linha 1 e a coluna 1 da matriz admitância podem ser desconsideradas formando uma matriz 9x9. Obtendo dessa forma o sistema linear (Figura 7).

Figura 6 - Sistema Elétrico de Potência 10 barras

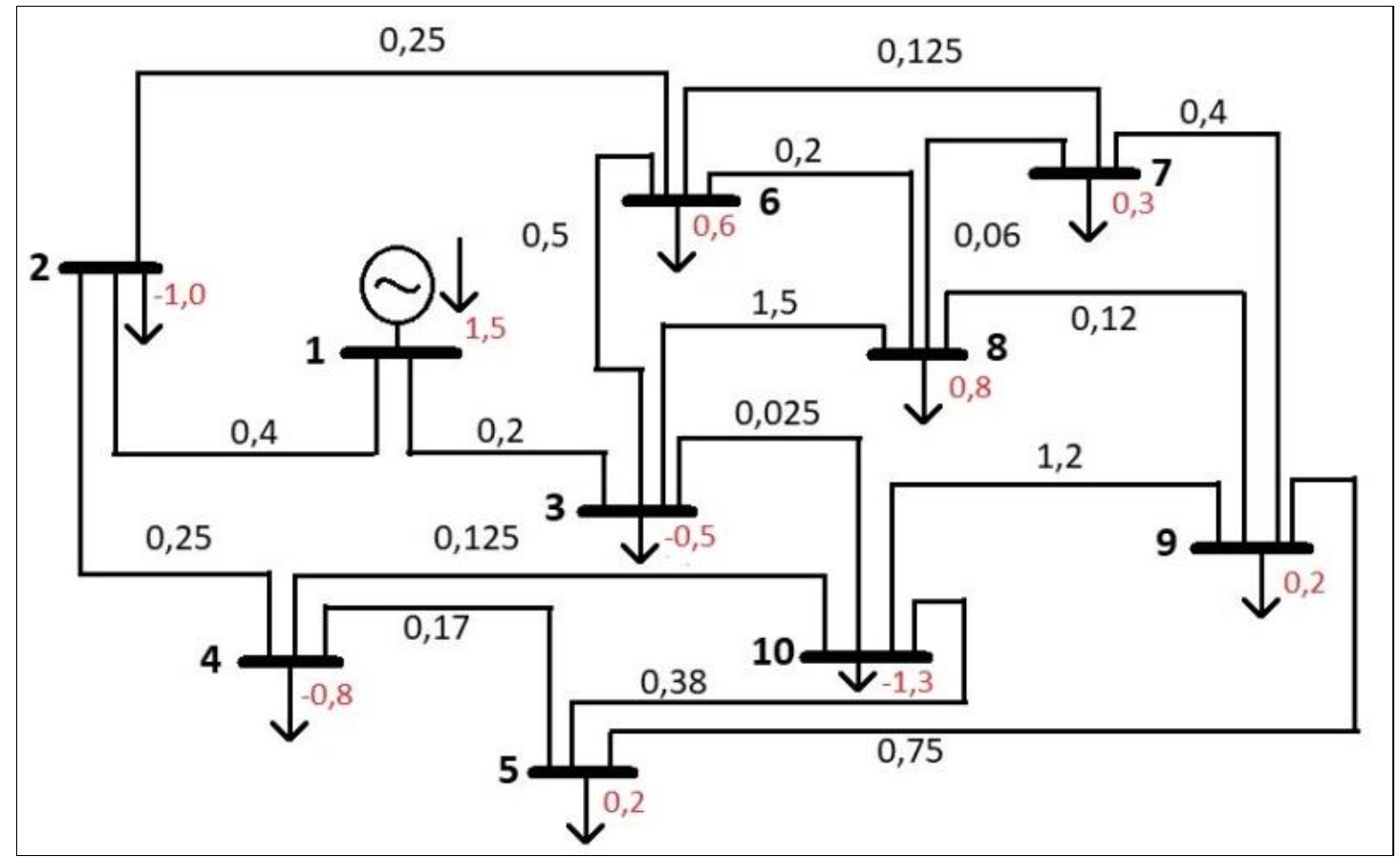

Figura 7 - Sistema linear 10 barras

\begin{tabular}{|c|c|c|c|c|c|c|c|c|c|c|c|c|}
\hline 10,5 & 0 & -4 & 0 & -4 & 0 & 0 & 0 & 0 & & & & -1 \\
\hline 0 & 47,666666 & 0 & 0 & -2 & 0 & $-0,6666666$ & 0 & -40 & & & & $-0,5$ \\
\hline-4 & 0 & 17,882353 & $-5,882353$ & 0 & 0 & 0 & 0 & -8 & & & & $-0,8$ \\
\hline 0 & 0 & $-5,882353$ & 9,847265 & 0 & 0 & 0 & $-1,333333$ & $-2,631579$ & & & & 0,2 \\
\hline-4 & -2 & 0 & 0 & 19 & -8 & -5 & 0 & 0 & & & & 0,6 \\
\hline 0 & 0 & 0 & 0 & -8 & 27,166666 & $-16,666666$ & $-2,5$ & 0 & & & & 0,3 \\
\hline 0 & $-0,666666$ & 0 & 0 & -5 & $-16,666666$ & 30,666666 & $-8,333333$ & 0 & & & & 0,8 \\
\hline 0 & 0 & 0 & $-1,333333$ & 0 & $-2,5$ & $-8,333333$ & 13 & $-0,833333$ & & & & 0,2 \\
\hline 0 & -40 & -8 & $-2,631579$ & 0 & 0 & 0 & $-0,833333$ & 51,464912 & & & & $-1,3$ \\
\hline
\end{tabular}

Os sistemas lineares gerados com 4 e 10 barras foram resolvidos com os métodos Eliminação de Gauss, Fatoração LU, Gauss Seidel e Crout. Todos implementados em linguagem de programação C e os resultados comparados com os obtidos pelo software MatLab. 


\section{Resultados e Discussão}

Verifica-se na Tabela 1 que os métodos Eliminação de Gauss, Fatoração LU e Método de Crout convergiram para o mesmo resultado, e apresentaram erro relativo ao software MatLab bem pequenos. Já o Gauss Seidel apresentou um erro relativo maior, em relação aos outros métodos, contudo ainda assim um erro relativo pequeno. A exatidão dos métodos implementados só não foi maior porque se usou apenas seis de mantissa.

Tabela 1 - Comparação dos Resultados 4 Barras

\begin{tabular}{c|c|c|l|c|c|c|c|c}
\hline MatLab & Gauss & Erro & Gauss Seidel & Erro & LU & Erro & Crout & Erro \\
\hline$-0,048233025492$ & $-0,048233$ & $2,55 \times 10^{-8}$ & $-0,04823$ & $1,03 \times 10^{-6}$ & $-0,048233$ & $2,55 \times 10^{-8}$ & $-0,048233$ & $2,55 \times 10^{-8}$ \\
\hline$-0,05405869272267$ & $-0,054059$ & $3,07 \times 10^{-7}$ & $-0,05405$ & $8,69 \times 10^{-6}$ & $-0,054059$ & $3,07 \times 10^{-7}$ & $-0,054059$ & $3,07 \times 10^{-7}$ \\
\hline$-0,0521938879704$ & $-0,052194$ & $1,12 \times 10^{-7}$ & $-0,05219$ & $4,89 \times 10^{-6}$ & $-0,052194$ & $1,12 \times 10^{-7}$ & $-0,052194$ & $1,12 \times 10^{-7}$ \\
\hline
\end{tabular}

A partir dos resultados apresentados na Tabela 1 é possível calcular o fluxo de potência ativa entre quaisquer barras do sistema de acordo com (1). A Tabela 2 apresenta a comparação do fluxo $\mathrm{P}_{23}$ entre a barra 2 e 3 com os resultados dos ângulos obtidos pelos métodos.

Tabela 2 - Fluxo de Potência $\mathrm{P}_{23} 4$ Barras.

\begin{tabular}{c|c|c|c|c}
\hline MatLab & Gauss & Gauss Seidel & LU & Crout \\
\hline$-0,0109694397192353$ & $-0,0109705882352941$ & $-0,0109411764705882$ & $-0,0109705882352941$ & $-0,0109705882352941$ \\
\hline
\end{tabular}

A Tabela 3 apresenta os resultados e erros relativos,do sistema gerado com 10 barras, obtidos pelos métodos Eliminação de Gauss, Gauss Seidel, Fatoração LU e Crout.

Tabela 3 - Comparação dos Resultados 10 Barras

\begin{tabular}{c|c|c|c|c|c|c|c|c}
\hline MatLab & Gauss & Erro & Gauss Seidel & Erro & LU & Erro & Crout & Erro \\
\hline$-0,189124094579$ & $-0,189124$ & $9,46 \times 10^{-8}$ & $-0,189112$ & $1,21 \times 10^{-5}$ & $-0,189124$ & $9,46 \times 10^{-8}$ & $-0,189124$ & $4,46 \times 10^{-8}$ \\
\hline$-0,205437977331$ & $-0,205438$ & $2,27 \times 10^{-8}$ & $-0,205426$ & $1,20 \times 10^{-5}$ & $-0,205438$ & $2,27 \times 10^{-8}$ & $-0,205438$ & $2,27 \times 10^{-8}$ \\
\hline$-0,254088959671$ & $-0,254089$ & $4,03 \times 10^{-8}$ & $-0,254076$ & $1,30 \times 10^{-5}$ & $-0,254089$ & $4,03 \times 10^{-8}$ & $-0,254089$ & $4,03 \times 10^{-8}$ \\
\hline$-0,190021945752$ & $-0,190022$ & $5,42 \times 10^{-8}$ & $-0,190008$ & $1,39 \times 10^{-5}$ & $-0,190022$ & $5,42 \times 10^{-8}$ & $-0,190022$ & $5,42 \times 10^{-8}$ \\
\hline 0,0076382114003 & 0,007638 & $2,11 \times 10^{-7}$ & 0,007655 & $1,68 \times 10^{-5}$ & 0,007638 & $2,11 \times 10^{-7}$ & 0,007638 & $2,11 \times 10^{-7}$ \\
\hline 0,0521625236884 & 0,052163 & $4,76 \times 10^{-7}$ & 0,052181 & $1,85 \times 10^{-5}$ & 0,052163 & $4,76 \times 10^{-7}$ & 0,052163 & $4,76 \times 10^{-7}$ \\
\hline 0,0590396320169 & 0,05904 & $3,68 \times 10^{-7}$ & 0,059057 & $1,74 \times 10^{-5}$ & 0,05904 & $3,68 \times 10^{-7}$ & 0,05904 & $3,68 \times 10^{-7}$ \\
\hline 0,0287929123547 & 0,028793 & $8,76 \times 10^{-8}$ & 0,02881 & $1,71 \times 10^{-5}$ & 0,028793 & $8,76 \times 10^{-8}$ & 0,028793 & $8,76 \times 10^{-8}$ \\
\hline$-0,233679491157$ & $-0,233679$ & $4,91 \times 10^{-7}$ & $-0,233667$ & $1,25 \times 10^{-5}$ & $-0,233679$ & $4,91 \times 10^{-7}$ & $-0,233679$ & $4,91 \times 10^{-7}$ \\
\hline
\end{tabular}

A Tabela 4 apresenta a comparação do fluxo $\mathrm{P}_{59}$ entre a barra 5 e 9 com os resultados dos ângulos obtidos pelos métodos.

Tabela 4 - Fluxo de Potência $\mathrm{P}_{59} 10$ Barras

\begin{tabular}{c|c|c|c|c}
\hline MatLab & Gauss & Gauss Seidel & LU & Crout \\
\hline$-0,291753144142267$ & $-0,291753333333333$ & $-0,291757333333333$ & $-0,291753333333333$ & $-0,291753333333333$ \\
\hline
\end{tabular}


Ao analisar a Tabela 3 em comparação com a Tabela 1, percebe-se que o tamanho do sistema não alterou a exatidão nos resultados dos métodos de Eliminação de Gauss, Fatoração LU e Crout. Em ambos os casos, tanto para o sistema 3x3 quanto para o sistema 9x9, esses métodos apresentaram erro relativo de ordem -7, -8. Já o método de Gauss Siedel apresentou um erro menor para o sistema $3 \times 3$, na ordem de -6 , e um erro maior para o sistema 9x9.

\section{Conclusão}

Os métodos numéricos para solução de sistemas lineares aqui aplicados permitem a análise e aumento da exatidão, e todos apresentaram um erro relativo bem pequeno, ou seja, os métodos se aproximaram do valor obtido pelo software MatLab, sendo que Eliminação de Gauss, Fatoração LU e Crout apresentaram menores erros relativos e não variaram a exatidão da resposta com o tamanho do sistema. O que não ocorreu com o Gauss Seidel que além de ter um erro maior apresentou variação de exatidão com o aumento do sistema.

Dessa forma é possível calcular o fluxo de potência ativa entre duas barras quaisquer no sistema de forma precisa e exata, o que contribui para uma análise correta de como o sistema está atuando.

O desenvolvimento do trabalho proporcionou aplicar e implementar métodos numéricos para solução de sistemas lineares em problemas da Engenharia Elétrica, apresentando soluções tão exatas quanto as obtidas por softwares matemáticos comerciais.

\section{Agradecimentos}

Os autores agradecem ao Programa FIPE/Sênior da UFSM pelo aporte financeiro no desenvolvimento das pesquisas realizadas.

\section{Referências}

MONTICELLI, Alcir José. Fluxo de carga em redes de energia elétrica. E. Blucher, 1983.

CHAPRA, Steven C.; CANALE, Raymond P. Métodos numéricos para engenharia. McGraw-Hill, 2008.

OLIVEIRA, Aurelio RL; SOARES FILHO, Secundino. Métodos de pontos interiores para problema de fluxo de potência ótimo DC. Sba: Controle \& Automação Sociedade Brasileira de Automática, v. 14, n. 3, p. 278-284, 2003.

BAPTISTA, Edméa Cássia; BELATt, Edmarcio Antônio; DA COSTA, Geraldo RM. Um método primal-dual aplicado na resolução do problema de fluxo de potência ótimo. Pesquisa Operacional, v. 24, n. 2, p. 215-226, 2004. 